\title{
Improving accuracy of nanothermal measurements via spatially distributed scanning thermal microscope probes
}

\author{
J. Spiece ${ }^{1}$, C. Evangeli ${ }^{1}$, K. Lulla ${ }^{1}$, A. Robson ${ }^{1}$, B. Robinson ${ }^{1,2}$ and O. Kolosov*1,2 \\ ${ }^{1}$ Physics Department, Lancaster University, LA1 4YB Lancaster, UK \\ ${ }^{2}$ Materials Science Institute, Lancaster University, LA1 4YW, Lancaster, UK. \\ *The correspondence to be addressed to: o.kolosov@lancaster.ac.uk
}

\section{Abstract}

Advances in materials design and device miniaturization lead to physical properties that may significantly differ from the bulk ones. In particular, thermal transport is strongly affected when the device dimensions approach the mean free path of heat carriers. Scanning Thermal Microscopy (SThM) is arguably the best approach for probing nanoscale thermal properties with few tens of nm lateral resolution. Typical SThM probes based on a microfabricated Pd resistive probes (PdRP) using a spatially distributed heater and a nanoscale tip in contact with the sample, provide high sensitivity and operation in ambient, vacuum and liquid environments. Whereas some aspects of the response of this sensor has been studied, both for static and dynamic measurements, here we build an analytical model of the PdRP sensor taking into account finite dimensions of the heater that improves the precision and stability of the quantitative measurements. In particular we analyse the probe response for heat flowing through a tip to the sample and due to probe self-heating and theoretically and experimentally demonstrate that they can differ by more than $50 \%$, hence introducing significant correction in the SThM measurements. Furthermore, we analyzed the effect of environmental parameters such as sample and microscope stage temperatures, and laser illumination, allowed to reduce the experimental scatter by a factor of 10 . Finally, varying these parameters, we measured 
absolute values of heat resistances and compared these to the model for both ambient and vacuum SThM operation, providing a comprehensive pathway improving the precision of the nanothermal measurements in SThM.

Keywords: nanoscale heat transfer; scanning thermal microscopy; precision thermal measurements; nanoscale thermal probes.

\section{Introduction}

Over the last two decades scanning probe microscopy has become an indispensable tool supporting developments in the nanoscience and nanotechnology thanks to its atomic-scale spatial resolution and sensitivity to a wide variety of physical properties. In particular, Scanning Thermal Microscopy (SThM) has enabled measurements of heat transport and temperatures at arbitrary selected points of the probed surface with the lateral resolution down to a few nm. SThM's outstanding performance is largely due to a range of nanofabricated probes that are both sensitive and easy to use. From biological applications to active semiconductor devices, SThM is becoming the ultimate tool for probing thermal properties at the nanoscale ${ }^{1-5}$. Among the variety of thermal probes developed, the commercially available family of Pd resistive probes (PdRP) $)^{6,7}$ based on microfabricated SiNx cantilevers is one of the most extensively used amongst the SThM community. The heater of PdRP also functions as a temperature sensing element with its position close to the sample surface and a nanoscale sharp tip apex. Combined with a good stability over time, this probe is efficient for both temperature and thermal conductance mapping in contact or semi-contact modes. Moreover, its main advantage is its ability to work in a range of conditions from vacuum ${ }^{8,9}$ and air $^{10}$ to liquids ${ }^{11}$. 
Very significantly, its ability to perform relevant measurements of thermal transport in ambient conditions, that has been a limiting factor for other SThM probes ${ }^{12}$, render PdRPs as the mainstream probe for nanothermal characterization, they have been used extensively for a variety of applications from biological studies ${ }^{13,14}$ to soft matter ${ }^{15}$ and condensed matter sciences $^{16-18}$.

However, merging the nanoscale resolution and high thermal sensitivity of a PdRP probe comes at a price - the heat flow through the nanoscale sized tip results in a temperature distribution which is detected by a thermal sensing element of several microns long. The modelling of this probe has been performed via finite elements analysis ${ }^{19}$ for static measurements and via unidirectional ${ }^{20}$ multipole ${ }^{21}$ and analytical models. These show that any analysis of sensor response must consider the heat transfer inside the spatially distributed heater and a highly heterogeneous probe, and corresponding sensor response have to be analyzed in order to allow precise nanothermal measurements, and to, ultimately, enable fully quantitative measurements of nanoscale thermophysical parameters. Here we will: derive a concise model with a minimal number of parameters to fully interpret the output of SThM measurements; compare these with experimental data of SThM operating in air and vacuum; and demonstrate the modification of the measurement setup allowing improvement of the stability, precision and quantitative interpretation of the measurements to be improved.

The typical PdRP sensor is composed of a $200 \mu \mathrm{m} \mathrm{Si} 3 \mathrm{~N}_{4}$ cantilever ending with a triangular beak-shaped tip of $14 \mu \mathrm{m}$ length. Two Pd lines ( $1 \mu \mathrm{m}$ wide $)$ are deposited on the sides of this triangular tip and joining at the tip apex (see Fig. S3). The sensor is included in a precision electrical circuit (usually a Wheatstone bridge) ${ }^{19}$ which allows both the measurement of the probes resistance, which is linearly dependent on the temperature averaged over the sensor 
area, and to apply elevated currents (up to $\sim 1 \mathrm{~mA}$ ) for Joule heating the sensor and hence the probe. An example of the effect of finite dimensions of the heater can be seen on Fig. 1a which shows the Finite Element Analysis (FEA) of the probe temperature distribution for the probe self-heating due to Joule self-heating in the Pd lines and Fig. 1b which shows the probe heated only due to the contact with the heated sample. In both cases the temperatures of the tip are identical. For the self-heated probe (Fig. 1a) the temperature distribution along the length of the triangular tip is approximately linear. At the same time, when heat is flowing through the tip apex, the temperature distribution changes drastically (Fig. 1b) heating predominantly the area around the apex. With the temperature sensing Pd lines extending along the whole length of the triangular tip, it is qualitatively clear that these temperature distributions will result in different probe resistances (and hence apparent probe temperatures) for the identical probe tip temperatures. The model developed in this paper allows us to account for this difference.
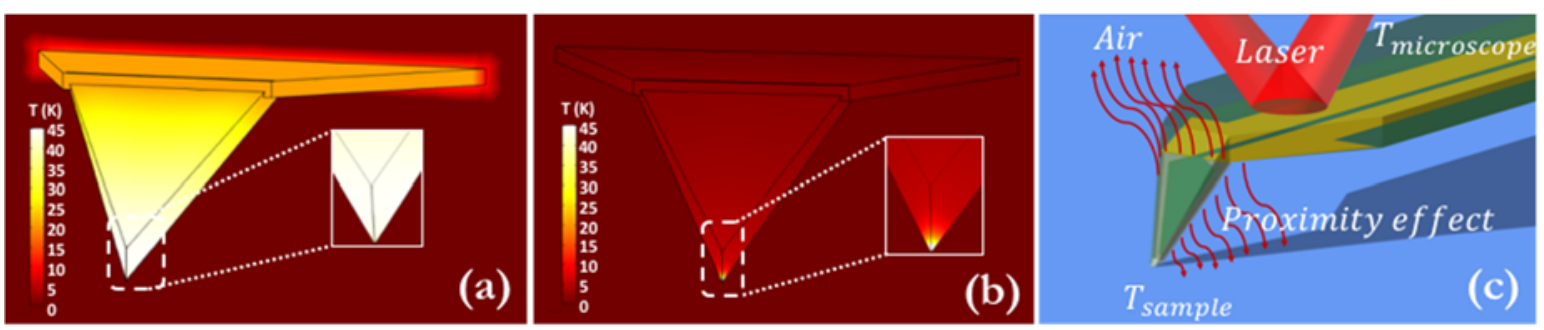

Fig. 1. Finite Element Analysis of (a) the self-heated probe and (b) the sample-heated probe with the same temperature of $45 \mathrm{~K}$ with respect to the background temperature (as represented by the color scale). (c) Schematic representation of the probe and the main source of uncertainty.

Here we also consider other experimental parameters which can influence SThM measurements during the experiment namely variations in sample temperature and microscope temperature, specifically where the base of the sensor is attached (Fig. 1c). These temperature variations - often referred to as "ambient temperature" - arise mainly from electrical effects arising from SThM operation, such as the feedback control of the position and laser 
illumination for positioning. In particular, the temperature variations due to laser illumination on the SThM probe are difficult to quantify ${ }^{22}$ as they vary depending on: i) the position of the laser beam on the cantilever which affects reproducibility of the measurements; ii) the laser source-cantilever distance which is not constant and thus causes variations of the probe temperature as it approaches the sample surface and iii) laser influenced artefacts arising from high topographical features and change of the sample reflectance. Indeed, in our experiments below, a rise of $0.5 \mathrm{~K}$ was typically observed over a 30 minutes timescale. Such variations present significant fraction of the probes temperature ${ }^{6}$, and even small variations of either of those temperatures can cause significant loss in the measurements reproducibility.

In order to tackle these challenges, we modified our custom-made SThM set-up with three elements:

- Monitoring of the sample and the probe mount (microscope) temperatures via high sensitivity calibrated thermistor temperature sensors to enable quantification of the thermal drift in the system and its correlation with the SThM signal variation.

- Active feedback stabilization of those two temperatures using Peltier modules mounted under the sample and on the probe holder.

- For the single-point measurements, we incorporated a second step in the measurements procedure - firstly measurement with the laser illumination to obtain a force curve and secondly without the laser illumination for thermal characterization.

These modifications, implemented below, resulted in a significant improvement in the precision and reproducibility of the measurements, in excellent correlation with our model of the SThM probe. 


\section{Theoretical and experimental methods}

\section{A. Analytical model of SThM sensor}

To allow quantitative SThM measurements, we have built a model assuming a thermal resistance formalism consistent with the system's physics (see Fig. 2a). The model is based on the regular triangular geometry of the probe and linear geometry of the Pd heater layer, as well as the environmental temperatures influencing the SThM measured signal. We solve the heat equation for the spatially distributed heater, with a finite number of input parameters, including power to the heater, temperatures of the sample, probe-holder and the temperature of air cell around the probe. The output of the model is the link between the tip-sample thermal resistance and "apparent" probe temperature measured via the change of the probe electrical resistance, and its dependence on the input parameters. As the characteristic dimensions of the probe and sample often lie in the transitional regime between ballistic and diffusive heat transport ${ }^{23}$, we have formulated measurements in terms of effective thermal resistances, similar to those reported $\mathrm{in}^{24}$. In Fig. 2a, the probe and tip are represented in green and the Pd film heater as two red strips. $T_{m}, T_{\text {air }}$ and $T_{s}$ are respectively microscope, ambient air and sample temperatures that act as the fixed-temperature heat-sinks for any heat flowing into the probe or generated in the probe heater, $Q_{h}$.

The significant dimensions of the Pd heater mean that the measured resistance is actually the electrical resistance averaged along the length of the heater. During the calibration procedure, the probe resistance $R_{e l}$ is linked to a calibration temperature $T_{c a l}$ by external and uniform heating ${ }^{19}$. The relation established is the following: $R_{e l} \propto \widehat{T_{h}}$ with $\widehat{T_{h}}$ the average heater temperature, $\widehat{T_{h}}=T_{c a l}$ in this case. For the self-heating, this average temperature differs from the temperature at the tip, $T_{t}$, due to the spatial distribution of the temperature on the heater 
(seen qualitatively in the Fig. 1a). This gives rise to a temperature distribution $T(l)$ in the heater. This distribution should depend on the geometrical and physical specificities of the heater as well as on the environment conditions.

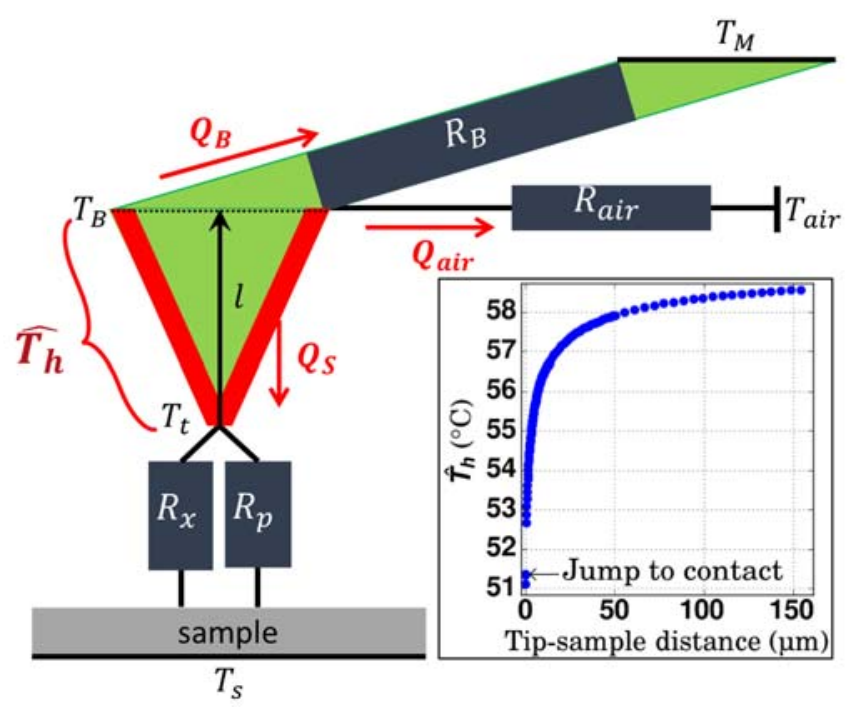

Fig. 2. Thermal resistance network with spatially distributed heater and measured probe temperature as a function of tip-sample surface distance. Sample, microscope and surrounding air heat baths are at temperatures $T_{S}, T_{M}$ and $T_{\text {air }}$, respectively. Heat produced $Q$ in the heater at the position $l$ is exchanged through 3 channels: $Q_{B}$ through the cantilever resistance $R_{B}, Q_{\text {air }}$ through the surrounding air resistance $R_{\text {air }}$ and $Q_{S}$ through the air gap proximity resistance $R_{p}$ and through the contact resistance $R_{x}$. The average heater temperature $\widehat{T}_{h}$ is obtained by averaging the temperature at each position $l$ between the tip apex temperature $T_{t}$ and the temperature at the cantilever junction $T_{B}$.

As the heater-sensor is distributed along the cantilever tip, the heat equation needs to describe a triangular system. First we consider the balance between the total heat generated in the heater and the different heat fluxes. Three heat channels are open: 1) through the cantilever base and its thermal resistance $R_{B}$, towards the microscope with temperature $T_{\mathrm{M}} ; 2$ ) through $R_{\text {air }}$ to the ambient environment with temperature $\left.T_{\text {air }} ; 3\right)$ to the sample with temperature $T_{\mathrm{s}}$ through both 
air proximity resistance $R_{p}$ and tip-sample contact resistance $R_{x}$ (where $\mathrm{R}_{\mathrm{x}}$ is only present when the direct contact between the tip and the surface is established). We therefore can write

$$
Q_{h}=\frac{T_{B}}{R_{B A}}-\frac{T_{M}}{R_{B}}-\frac{T_{a i r}}{R_{a i r}}+\frac{T_{t}}{R_{x p}}-\frac{T_{S}}{R_{x p}}
$$

where $\frac{1}{R_{B A}}=\frac{1}{R_{B}}+\frac{1}{R_{\text {air }}}$ and $\frac{1}{R_{x p}}=\frac{1}{R_{x}}+\frac{1}{R_{p}}$.

It should be noted that the proximity air resistance is strongly dependent on the tip-surface distance in the range of 10-15 $\mu \mathrm{m}$ (as measurement shown in Fig. 2 inset) - the same order as the dimensions of the triangular part of the PdRP probe.

To see the main features of this model, we first consider the system in vacuum where $R_{\text {air }}=$ $R_{p}=\infty$. The linear shape of the heater leads to the equal distribution of the Joule heat produced along the heater length. Due to the symmetry of the system, and the direction of the heat propagation along the axis of the probe, we can establish a link between $T_{B}$ and $T_{t}$ as

$$
T_{B}-T_{t}=a Q_{h}\left(\frac{\ln (1+b)}{b}-1\right)+\frac{a\left(T_{t}-T_{S}\right) \ln (1+b)}{R_{x p}}
$$

where $b=\frac{l_{h}}{l_{t}}$ and $l_{h}$ is the heater length and $l_{t}$ is the length of the truncated tip and $a=\frac{l_{h}+l_{t}}{2 h k r_{b}}$ such that $h, k$ and $r_{b}$ are the heater thickness, effective thermal conductivity and radius at the cantilever junction, respectively (See Supplementary Materials S3 for details).

From Eq. 1 and 2, we can write the temperature distribution in the heater as:

$$
T_{h}(l)=T_{t}-\frac{a Q_{h}\left(l-l_{t}\right)}{b l_{t}}+a\left(\frac{Q_{h}}{b}+\frac{T_{t}-T_{S}}{R_{x p}}\right) \ln \frac{l}{l_{t}}
$$

Finally, in order to obtain the average heater temperature which is the parameter measured during experiment, we average this distribution over the heater length: 


$$
\widehat{T_{h}}=\frac{1}{l_{h}} \int_{l_{t}}^{l_{t}+l_{h}} T_{h}(l) d l
$$

Eq. 3 shows that the temperature distribution due to the self-heating is linear along the probe's (second term in Eq. 3), whereas the temperature distribution in the probe due to the heat produced by the tip (third term in Eq. 3) has a non-linear logarithmic dependence. Together Eqs. 3 and 4 provide the temperature of the probe as the function of the probe-sample resistance. This resistance is the ultimate parameter to be measured in SThM experiments.

\section{B. High precision SThM set up}

In order to compare experimental data with our model, we first need to address several common sources of error that affect PdRP as well as any other SThM probes. We now report an experimental setup and measurement sequence which improves the stability and the precision of SThM measurement. A detailed description of the experimental setup is available in the Supplementary Materials Section S1.

A set of typical electrical signals measured in an SThM are shown in Fig. 3 for ambient (a) and vacuum (b) conditions. The electrical SThM signal (voltage) is proportional to the resistance of the self-heated probe and is recorded as the probe approaches to and then retracts from the sample surface. When the probes snaps in and solid-solid contact is established, a sharp drop is observed linked with the increase heat transport through the $R_{x}$ channel (Fig. 2). During retraction, adhesion forces require an increased negative force to rupture the contact, hence the approach and retract curves do not overlap, creating hysteresis. In air, adhesion forces are higher due, mainly, to the liquid meniscus; the increased hysteresis in air can be clearly 
observed when comparing air and vacuum curves. The voltage is recorded immediately prior to probe-sample contact $V_{n c}$ then as again in contact $V_{c}$. From these two voltages, we extract the SThM response $d V / V$ where $\frac{d V}{V}=\frac{V_{n c}-V_{c}}{V_{n c}}$.

Whilst, from the model developed above, it can be shown that $d V / V$ depends most strongly on the tip sample heat transfer, the laser illumination on the back of the cantilever can drastically affect the SThM signals (as discussed in the Introduction). The inset of Fig. 3 shows SThM responses for different laser positions on the probe, different laser alignments lead to a data scatter error of up to $30 \%$ of the absolute measured value. Additionally, the laser illumination can cause interference with the electrical signals affecting the measurement precision (see Fig. S1). Therefore, as initially observed by our group and later confirmed elsewhere ${ }^{22}$, conducting measurements in absence of laser can greatly improve the stability of SThM measurements. In all experiments reported below, the laser was shut off. 


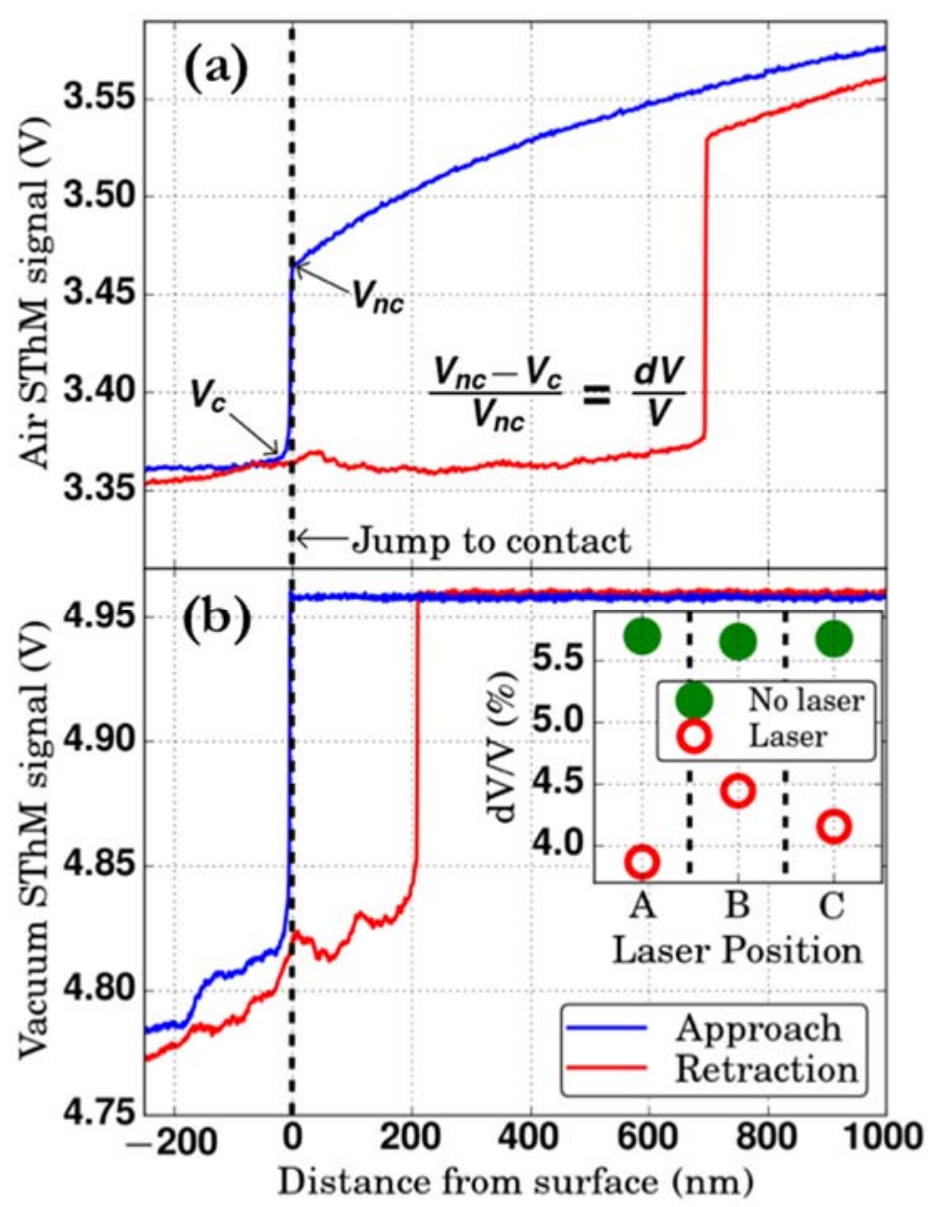

Fig. 3. Approach (blue) - Retraction (red) SThM curves acquired with PdRP in ambient (a) and vacuum (b) conditions on a $\mathrm{SiO}_{2} / \mathrm{Si}$ sample. Inset: Thermal response $(\mathrm{dV} / \mathrm{V})$ obtained when aligning the laser at 3 positions $(\mathrm{A}, \mathrm{B}, \mathrm{C})$ and compared to response without laser.

Fig. 4 shows the effect of the sample and probe base temperatures on the reproducibility of the SThM response. We performed 240 consecutive approach-retraction cycles on a Si sample at the same point while monitoring $T_{S}$ and $T_{M}$. The SThM signals, corresponding to $\widehat{T}_{h}$ before (open circles, Fig. 4b) and after the contact (closed circles, Fig. 4b) were measured simultaneously with those of the sample (open circles, Fig. 4a) and microscope temperature (closed circles, Fig. 4a). One can clearly observe that the thermal signals in- and out-of-contact are changing with time in a similar manner to the sample and microscope temperatures confirming the model conclusion in Eq. 3. 


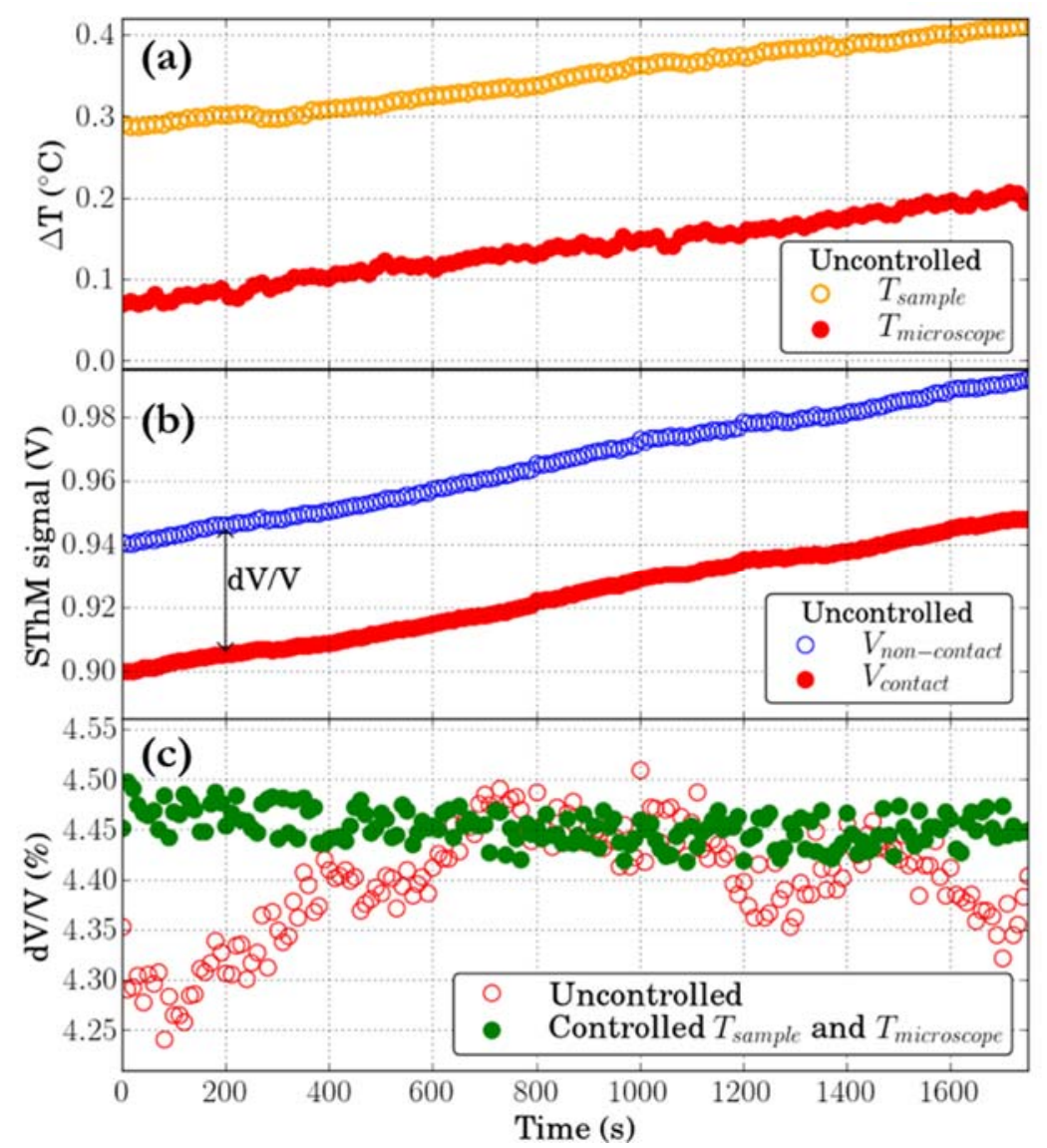

Fig. 4. (a) Temperature of the microscope (red filled circles) and sample (yellow open circles) vs time and (b) the corresponding measured SThM signal (excess probe temperature) (V) for tip out of contact (blue open circles) and in-contact (red filled circles) (sample and microscope temperatures are uncontrolled). (c) Thermal response (dV/V) acquired, from consecutive point contacts, at controlled (constant sample-microscope temperature) (green filled circles) vs uncontrolled (red open circles) sample temperature.

During an experiment, the thermal response is expected to be constant at the same point of a sample, when taking consecutive measurements. Instead, in the SThM setup with uncontrolled sample and probe base temperatures, we observe that the thermal response varied with time (see Fig. 4c, open circles). To compensate for this temperature drift, we used Peltier plates mounted underneath the sample and on the probe holder, enabling a close loop control of temperatures $T_{S}, T_{M}$ via thermistors attached to the sample and the probe base. The repeated 
measurements in this setup (Fig. 4c, filled green points) showed about 5-10 fold stability increase over the same measurement time. These results indicate that keeping the sample and probe holder temperatures constant leads to significantly more reproducible SThM measurements.

\section{Results and discussion}

\section{A. Experimentally measured model parameters}

In order to measure accurately the main thermal resistances of the model, a specific procedure needs to be followed. First, we place the probe in vacuum. In this condition, $\widehat{T_{h}}$ is given by

$$
\widehat{T_{h}}=\left(R_{B}+\gamma_{s h}\right) Q_{h}+T_{m}
$$

where $\gamma_{s h}=\frac{l_{h}+l_{t}}{2 h k r_{b} l_{h}}\left[\frac{l_{h}}{2}+\frac{l_{t}^{2}}{l_{h}} \ln \left(\frac{l_{t}+l_{h}}{l_{t}}\right)-l_{t}\right]$. Then, knowing the power $Q_{h}$ injected in the probe and the temperature difference $\widehat{T_{h}}-T_{m}$, we can measure the probe total thermal resistance $R_{B}+\gamma_{s h}$. The contribution of the triangular tip is often neglected in the literature ${ }^{25-}$ ${ }^{27}$ and values reported for the probe thermal resistance just take into account $R_{B}$. Our approach therefore gives a more precise understanding of the heat transfer within the probe.

Decoupling $R_{B}$ from $\gamma_{s h}$ is difficult experimentally. However, FEA modelling previously reported $^{19}$, or analytical models such as the one proposed by Ge et al. ${ }^{28}$ show that $R_{B}$ accounts for around 25 to $50 \%$ of the total thermal resistance measured and $\gamma_{s h}$ for the rest. We measured a total probe thermal resistance $R_{B}+\gamma_{s h}=7 \pm 0.5 \times 10^{4} \mathrm{KW}^{-1}$ which gives $R_{B}=1.8 \pm$ $0.1 \times 10^{4} \mathrm{KW}^{-1}$ and $\gamma_{s h}=5.3 \pm 0.4 \times 10^{4} \mathrm{KW}^{-1}$ assuming $\frac{R_{B}}{R_{B}+\gamma_{s h}}=0.25$ 
In air and far away from the sample surface, we have

$$
\widehat{T_{h}}=T_{m} \frac{R_{\text {air }}}{R_{B}+R_{\text {air }}}+T_{\text {air }} \frac{R_{B}}{R_{B}+R_{\text {air }}}+Q_{h}\left[\gamma_{s h}+\frac{R_{B} R_{\text {air }}}{R_{B}+R_{\text {air }}}\right]
$$

As we deduced $R_{B}$ and $\gamma_{s h}$ from the previous step, we can obtain $R_{\text {air }}$ if we know or assume $T_{\text {air. }}$. We obtained $R_{\text {air }}=5 \pm 1 \times 10^{5} \mathrm{KW}^{-1}$.

Finally, if we move the probe toward the sample surface, we notice a drop in the probe average temperature $\widehat{T_{h}}$ (see Fig. 2 b). This arises from the heat conductance through the air gap, which we called $R_{p}$. Comparing the signal far away from the surface to the one just before snap-in, we can measure $R_{p}$ for a given power $Q_{h}$ (equations are given in the Supplementary Material section S6). As we will discuss in the last section, the proximity effect plays a strong role in measurements performed in air. Measured values for the model parameters $R_{B}, \gamma_{s h}, R_{a i r}$ and $R_{p}$ are given in Table I.

The final expression for the average heater temperature in air and in contact with the sample is given by:

$$
\widehat{T_{h}}=\alpha Q_{h}+\beta T_{m}+\gamma T_{s}+\delta T_{a i r}
$$

where coefficients $\alpha, \beta, \gamma$ and $\delta$ are functions of $R_{B}, R_{a i r}, R_{p}, R_{X}$ and $l_{h}, l_{t}$ as defined above, which are physical and geometrical parameters of the probe. Full expressions for $\widehat{T_{h}}$ and $\alpha, \beta, \gamma$ and $\delta$ are given in the Supplementary Materials in Section S3.

The key consequence of the model summarized in Eq. 7, as opposed to simpler models, is that it links the key value measured in SThM ("measurand") $\widehat{T}_{h}$ with environmental temperatures 
$T_{m}, T_{s}, T_{\text {air }}$ and heat (Joule heating in the probe) $Q_{h}$, and clearly confirms the linear dependence on them. The proposed calibration protocol - cantilever and heater resistances calibration in vacuum, air resistance calibration far away from the sample and proximity resistance just before contact - allows the quantification of all parameters of the thermal model shown on Fig. 1 except the contact resistance $R_{X}$. The extraction of this ultimate parameter can be realized by inverting Eq. 7 and expressing $R_{X}$ as a function of the measured $\widehat{T}_{h}$ and the rest of the parameters.

Table I. Measured nominal values of the model parameters used to extract thermal contact resistance $R_{X}$.

\begin{tabular}{|c|c|}
\hline Parameter & Measured value \\
\hline$R_{B}$ & $1.75 \pm 0.08 \times 10^{4} \mathrm{KW}^{-1}$ \\
\hline$\gamma_{s h}$ & $5.25 \pm 0.08 \times 10^{4} \mathrm{KW}^{-1}$ \\
\hline$R_{\text {air }}$ & $5 \pm 1 \times 10^{5} \mathrm{KW}^{-1}$ \\
\hline$R_{p}$ & $7 \pm 1 \times 10^{5} \mathrm{KW}^{-1}$ \\
\hline
\end{tabular}

The essential feature of the model developed above is that it accounts for the probe geometry. As the heater is distributed on the triangular tip, a temperature gradient is inevitably formed. This gradient is different in and out of contact. It is also different depending on whether the heat is generated within the probe (self-heating case) or is coming from the sample through the tip. In Fig. 5a, temperature distributions are displayed for both cases, self-heated tip and heat flowing from the sample. The distributions are drastically different. Note that in Fig. 5a we compared Finite Element modelling to our analytical model (Eq. 3) and found similar trend which supports the analytical model presented in this paper. 
Furthermore, a key finding of our model is the comparison of heat fluxes to the self-heated probe and to the sample. When power is provided to the heater via the Pd film (self-heating), the temperature rises with $\frac{d \widehat{T_{h}}}{d Q_{h}}=20.5 \pm 0.5 \mathrm{Km}^{-1} \mathrm{~W}^{-1}$. However, when the same amount of heat is sent through the tip apex, the temperature rise is significantly higher than the selfheating one. In this case, the temperature increases with $\frac{d \widehat{T_{h}}}{d Q_{S}}=31.9 \pm 0.5 \mathrm{Km}^{-1} \mathrm{~W}^{-1}$. This fundamental difference of more than $50 \%$, is often overlooked in the interpretation of SThM results. Thus, a model taking into account the dimensions of the heater is absolutely essential for the correct interpretation of the measurement results.

As we mentioned earlier, in the non-contact case and far away from the sample, the tip temperature $T_{t}$ is always higher than the average heater temperature $\widehat{T_{h}}$. In Fig. $5 \mathrm{~b}$, we compute and compare these two temperatures. For the same electrical reading, e.g. electrical resistance, two different temperatures are obtained (detailed curves are provided in Supplementary Materials, S5). Importantly, self-heating and tip temperature differ by more than $40 \%$. These model results highlight the importance of taking into account the heater finite dimensions in order to extract physical quantities of the SThM measurement.

Principally, the spatially distributed probe increased response to the tip heating (by about 50\%) and concentration of the temperature rise to the probe apex, provides quantitative basis to the reported efficiency of such SThM probe in ambient ${ }^{19}$ as well liquid immersion (iSThM) ${ }^{11}$ thermal measurements. The tip weighted sensitivity and self-heating make it superior for both local temperature and thermal conductivity measurements compared to the compared to the silicon microfabricated probes ${ }^{29}$ that while providing somewhat better spatial resolution (down to $10 \mathrm{~nm}$ ) can effectively operate as thermal probe mainly in the vacuum environments. 
(a)

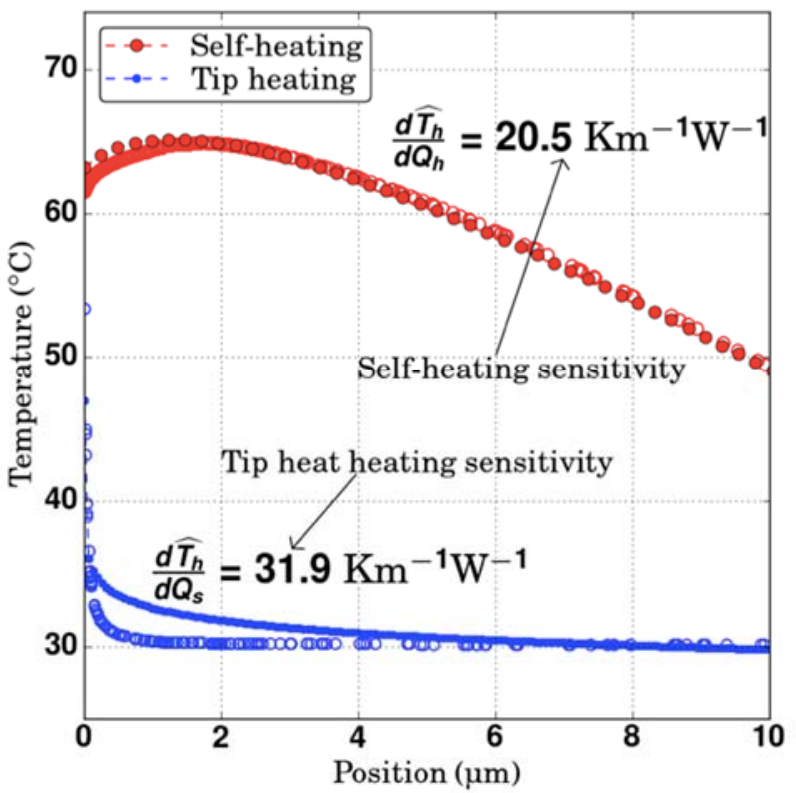

(b)

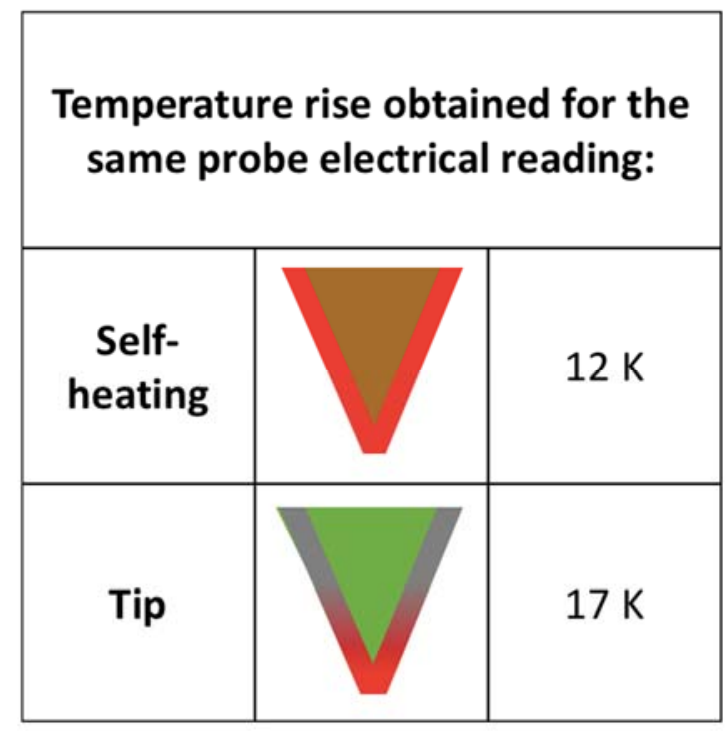

Fig. 5. (a) Heater temperature distribution for the self-heated case (top curve - red circles) and due to heat flow through the tip (bottom curve - blue circles) as obtained from finite element analysis (open circles) and analytical model (filled circles). (b) Values of computed temperature rise of the probe tip obtained for the same electrical reading of the probe: color of the heater at the edge of the probe schematically represents temperature distribution in the probe. The two temperatures are often considered to be the same.

\section{B. Model outputs vs multiparametric experiments}

We performed experiments in different conditions in order to compare our model with experimental data. A set of SThM measurements on a single crystalline diamond sample (Element Six, UK) were realized. Diamond was chosen for its high thermal conductivity above $1000 \mathrm{Wm}^{-1} \mathrm{~K}^{-1}$ and therefore the main component of the thermal response will be the contact resistance at the probe apex leading to minimal effect of the sample itself. The SThM response $d V / V$ for different powers was measured for 3 sample temperatures from below to above the microscope temperature. This experiment was performed both in vacuum and in air 
environments. Model parameters were then changed to correspond to experimental conditions and measurements and model outputs compared (Fig. 6a,b).

Model results are plotted in solid lines. As we see at Fig. 6a, a linear trend is obtained in vacuum for both model and experiment. Noticeably, when the microscope and sample temperatures are equal, the SThM response does not depend on the power injected in the heater. Such conditions (vacuum and equal sample and microscope temperatures) provide the best system for precise and repeatable SThM measurements.

In air, the situation differs drastically (see Fig. 6b). The proximity effect is playing a key role through the air gap resistance between the heater and the sample surface. To account for this effect, we changed the value of the air gap resistance for the different powers. It is likely that different probe temperatures lead to different proximity resistances. We assumed inversely proportional air gap resistance with input power. As we can see in Fig. 6b, when microscope and sample temperatures are equal, the SThM response splits with a variation of $0.5 \%$. Finally, we also notice that our model does not account fully for this proximity effect as from the experimental data non-linear effects are observed and could not be reproduced numerically. This result highlights the importance of proximity effect in ambient SThM measurements. 

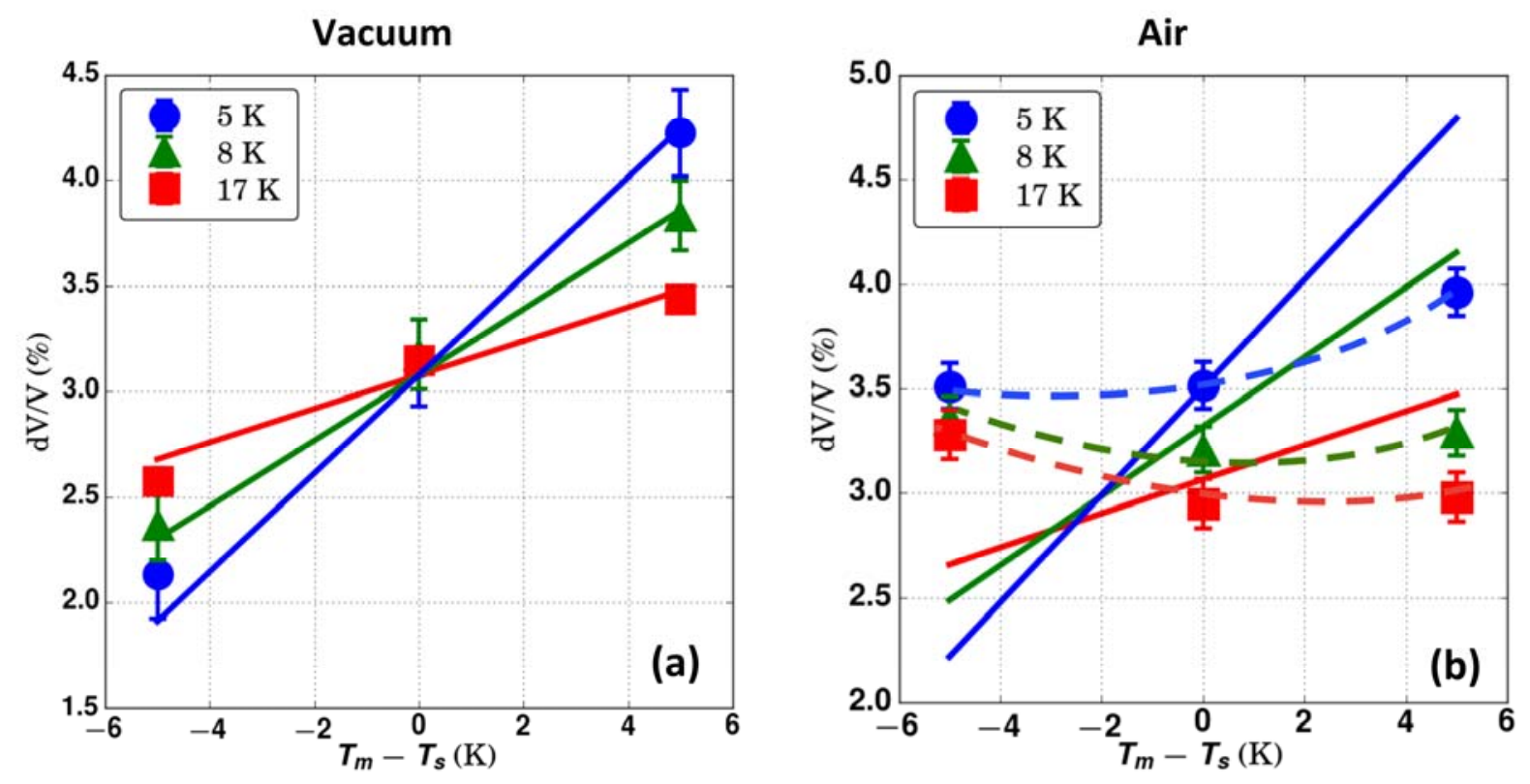

Fig. 6. Experimental (solid circles) and model (solid lines) results for the thermal response $d V / V$ as a function of sample temperature in vacuum (left) and in air (right) for three average heater excess temperature ( $5 \mathrm{~K}, 8 \mathrm{~K}$ and $17 \mathrm{~K})$. Dashed lines in panel (b) are a guide for the eye.

\section{Conclusions}

This study explored a sensitivity of SThM based on the most widely used commercial metal resistive probe capable to operate in air, vacuum and liquid environments. By building a simple semi-analytical model, we demonstrated the importance of taking into account the finite dimensions of the SThM sensor. Both our model and experiments show that the self-heating of the probe and heating through the tip apex with the same amount of heat result in the very different response of the probe, as large as $50 \%$ for a typical probe, the fact essential to be taken into account in any quantitative SThM measurements. Furthermore, crucial parameters impacting the experimental results were found - namely influence of laser providing position feedback in typical SThM setup, and small variations of the sample and microscope 
temperatures, often assumed to be equal, were shown to significantly affect the measurements reproducibility and precision.

By adding Peltier temperature control of the sample and the probe holder, we substantially decreased data scatter from $5.5 \%$ to $0.5 \%$. Allowing two step measurements - with laser to monitor the sample topography, and blocking the laser for SThM measurements - significantly improved the SThM data scatter that otherwise can add up to $30 \%$ to the data scatter. When compared to experiments, we obtained good agreement with our model when the system is under vacuum. In air, we could partially reproduce the experiment by changing the proximity thermal resistance in the model. This results shows the importance of the proximity effect in scanning thermal microscopy measurements which requires more precise modelling.

Both the modelling and experimental aspects of this study allow up to order of magnitude improvement in precision of current scanning thermal microscopy techniques. The increased precision and interpretation of the SThM measurements may provide significant insight into the mainstream semiconductor sector looking for nanoscale heat dissipation in advanced processors, novel nanostructured thermoelectrics and thermal interface materials.

Supplementary Materials: detailed description of the experimental setup and SEM image of the probe; influence of laser in the SThM; derivation of the average heater temperature; probe response for calibration, self-heating and tip heating.

Acknowledgements. Authors acknowledge scientific discussions with S. Gomès, OVK acknowledges the support of project EU FP7-NMP-2013-LARGE-7 QUANTIHEAT 
(604668), EPSRC grant EP/K023373/1 and EP/G015570/1, Paul Instrument Fund (c/o) The

Royal Society, as well as support of Lancaster University Impact Acceleration Account.

$1 \quad$ S. Gomès, A. Assy, and P.-O. Chapuis, physica status solidi (a) 212, 477 (2015).

2 D. G. Cahill, W. K. Ford, K. E. Goodson, G. D. Mahan, A. Majumdar, H. J. Maris, R. Merlin, and S. R. Phillpot, Journal of Applied Physics 93, 793 (2003). M. M. Kim, A. Giry, M. Mastiani, G. O. Rodrigues, A. Reis, and P. Mandin, Microelectronic Engineering 148, 129 (2015).

A. Majumdar, Annual Review of Materials Science 29, 505 (1999).

L. Shi and A. Majumdar, Journal of Heat Transfer-Transactions of the Asme 124, 329 (2002).

P. S. Dobson, J. M. R. Weaver, G. Mills, and leee, in 2007 leee Sensors, Vols 1-3 (leee, New York, 2007), p. 708. G. Mills, H. Zhou, A. Midha, L. Donaldson, and J. M. R. Weaver, Applied Physics Letters 72, 2900 (1998). M. E. Pumarol, M. C. Rosamond, P. Tovee, M. C. Petty, D. A. Zeze, V. Falko, and O. V. Kolosov, Nano Letters 12, 2906 (2012). M. Hinz, O. Marti, B. Gotsmann, M. A. Lantz, and U. Durig, Applied Physics Letters 92, 3 (2008). Y. Zhang, P. S. Dobson, and J. M. R. Weaver, Journal of Vacuum Science \& Technology B 30 (2012).

P. D. Tovee and O. V. Kolosov, Nanotechnology 24, 465706 (2013).

N. D. Masters, W. Ye, and W. P. King, Physics of Fluids 17, 100615 (2005).

L. Mu, Y. Li, N. Mehra, T. Ji, and J. Zhu, ACS Appl Mater Interfaces 9, 12138 (2017).

D. Xu, Y. Zhang, H. Zhou, Y. Meng, and S. Wang, Holzforschung 70 (2016).

A. Dawson, M. Rides, A. S. Maxwell, A. Cuenat, and A. R. Samano, Polymer Testing 41, 198 (2015).

S. Crossley, T. Usui, B. Nair, S. Kar-Narayan, X. Moya, S. Hirose, A. Ando, and N. D. Mathur, Applied Physics Letters 108, 032902 (2016). T. Gotszalk, and P. Grabiec, Micron 79, 93 (2015).

K. W. Park, E. M. Krivoy, H. P. Nair, S. R. Bank, and E. T. Yu, Nanotechnology 26, 265701 (2015).

P. Tovee, M. Pumarol, D. Zeze, K. Kjoller, and O. Kolosov, Journal of Applied Physics 112, 114317 (2012).

E. Puyoo, S. Grauby, J. M. Rampnoux, E. Rouviere, and S. Dilhaire, Journal of Applied Physics 109, 9 (2011).

J. Bodzenta, M. Chirtoc, and J. Juszczyk, Journal of Applied Physics 116, 054501 (2014).

L. Ramiandrisoa, A. Allard, Y. Joumani, B. Hay, and S. Gomés, Review of Scientific Instruments 88, 125115 (2017).

G. Chen, International Journal of Thermal Sciences 39, 471 (2000).

N. Maxim, C. R. Mark, J. G. Andrew, V. K. Oleg, G. D. Vladimir, and A. Z. Dagou, Journal of Physics D: Applied Physics 50, 494004 (2017). 
A. Assy and S. Gomès, Applied Physics Letters 107, 043105 (2015).

A. Kaźmierczak-Bałata, J. Juszczyk, D. Trefon-Radziejewska, and J. Bodzenta, Journal of Applied Physics 121, 114502 (2017). J. L. Battaglia, A. Saci, I. De, R. Cecchini, S. Selmo, M. Fanciulli, S. Cecchi, and M. Longo, physica status solidi (a) 214, 1600500 (2017). Y. Ge, Y. Zhang, J. A. Booth, J. M. Weaver, and P. S. Dobson, Nanotechnology 27, 325503 (2016).

B. A. Nelson and W. P. King, Review of Scientific Instruments 78, 023702 (2007). 


\title{
Supplementary Materials
}

\section{Improving accuracy of nanothermal measurements via spatially distributed scanning thermal microscope probes}

\author{
J. Spiece ${ }^{1}$, C. Evangeli ${ }^{1}$, K. Lulla $^{1}$, A. Robson ${ }^{1}$, B. Robinson ${ }^{1,2}$ and O. Kolosov*1,2 \\ ${ }^{1}$ Physics department, Lancaster University, LA1 4YB Lancaster, UK \\ ${ }^{1}$ Materials Science Institute, Lancaster University, LA1 4YW, Lancaster, UK. \\ *The correspondence to be addressed to: o.kolosov@lancaster.ac.uk \\ WWW: www.nano-science.com
}

\section{S1. Experimental set-up description}

All experiments were performed using a high vacuum multifunctional SPM (HV NT-MDT Solver HV-AFM) either at ambient pressure or in vacuum ( $10^{-7}$ Torr). SThM probes were provided by Windsor Scientific (KNT-SThM-1an) and mounted on a half-moon washer with metallic support for magnetic attachment on the AFM probe holder. The probe was then connected through a modified Wheastone bridge and biased by an AC+DC voltage. We used a function generator (Keithley $339050 \mathrm{MHz}$ arbitrary waveform generator) as the voltage source. The bridge resistors and capacitors are balanced at room temperature while supplying low power $\left(<10^{-7} \mathrm{~W}\right)$ on the probe and in the absence of laser illumination in order to avoid any self-heating or excess temperature on the heater. The bridge output voltage is fed into a lock-in amplifier (SRS-830, Stanford Research Systems). This allows us to quantify directly the self-heating of the probe with the different voltages applied.

Before performing experiments, the probe resistance was calibrated on a hot plate (Torrey Pines Scientific, Echo Therm model IC20). Varying the plate temperature and the DC voltage supplied to the probe, we used a dedicated multimeter (Agilent 34401A 6.5 digits precision) in the ratiometric mode. This procedure allows us to measure the probe electrical resistance as a function of the voltage and temperature. Therefore, the self-heating, or Joule heating, of 
the probe could also be measured precisely and the probe excess temperature is obtained as a function of the power supplied.

\section{S2. Force-Spectroscopy SThM and laser influence}

In standard AFM Force-Spectroscopy, the laser deflection on the cantilever is related to the spring constant of both probe and sample. If the same procedure is applied with an SThM probe, both force and thermal signal can be recorded simultaneously as on Fig. S1. However, the laser induces interferences which can be seen in both deflection and SThM signals.

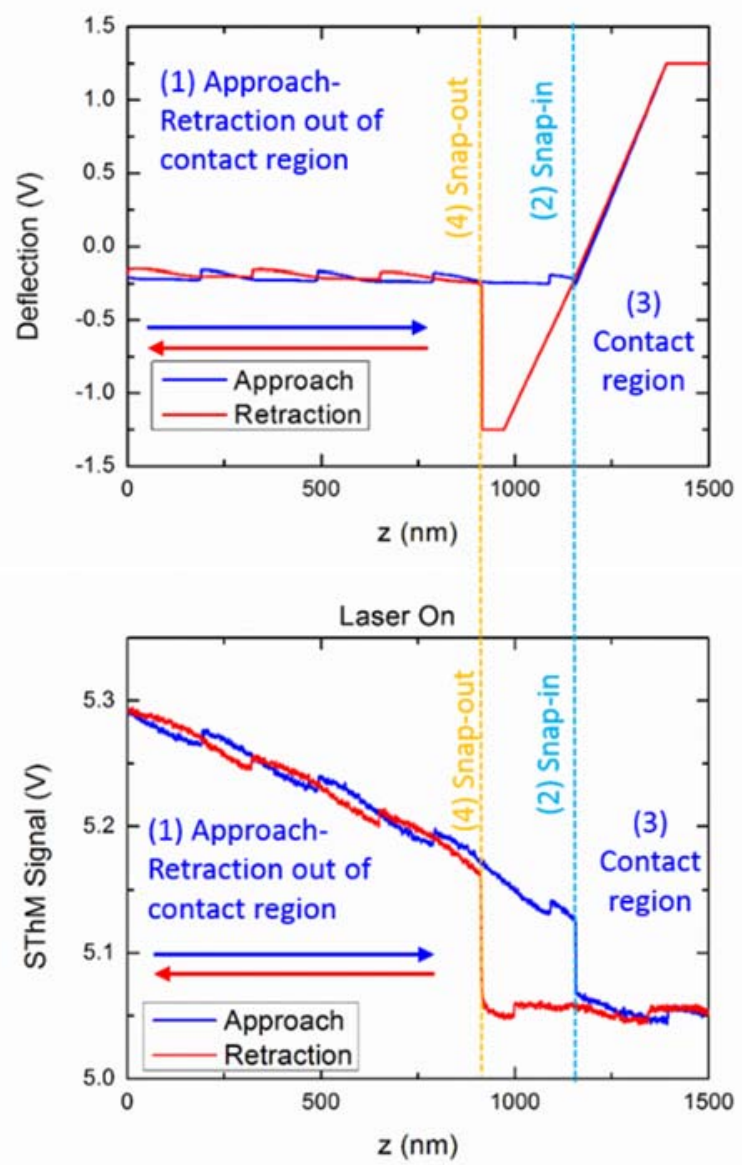

Fig. S1. (a) Approach (blue) - Retraction (a) force curve (top) and FS-SThM curve (b) acquired simultaneously with PdRP in ambient conditions on a $\mathrm{Al}_{2} \mathrm{O}_{3} / \mathrm{Si}$ sample $\left(T_{\text {probe }}=350 \mathrm{~K}, T_{\text {base }}=T_{\text {sample }}=300\right.$ K).

We measured the thermal response as a function of the laser position on the cantilever (Fig. S2) and compared it to the response without laser. We observed a strong influence of the laser on the measurement outputs. Variations as large as $12 \%$ were observed. 


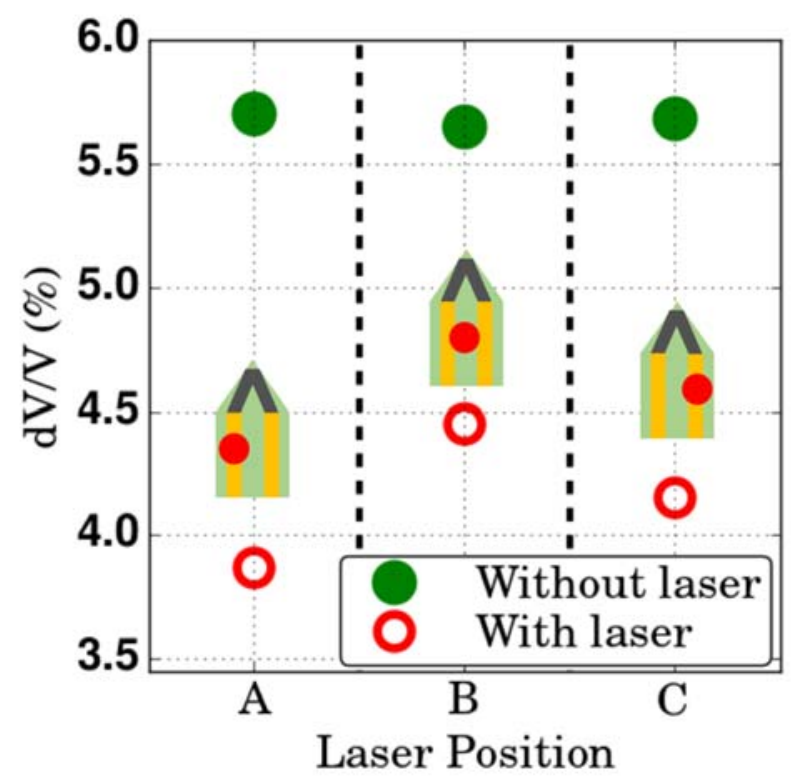

Fig. S2. Thermal response $(d V / V)$ obtained when aligning the laser at 3 positions $(A, B, C)$ and compared to response without laser.

S3. Final expression for the average heater temperature in contact

The final expression for the average heater temperature in air and in contact with the sample is given by:

$$
\widehat{T_{h}}=\alpha Q_{h}+\beta T_{m}+\gamma T_{s}+\delta T_{\text {air }}
$$

with 


$$
\begin{aligned}
& \alpha=\frac{1}{\Omega}\left(l_{h}\left(R_{x}+R_{p}\right)\left(R_{b}+R_{a}\right)\left(l_{h}+2 l_{t}\right) a+\left(2\left(R_{x}+R_{p}\right)\right) R_{B} R_{a} l_{h}^{2}\right. \\
& +\left(4\left(R_{x}+R_{p}\right)\right) R_{b} R_{a} l_{t} l_{h} \\
& \left.+\left(2\left(\left(\left(R_{x}+R_{p}\right) R_{b}+R_{x} R_{p}\right) R_{a}+R_{x} R_{p} R_{b}\right)\right) l t^{2}\right) a \ln \left(\frac{l_{t}+l_{h}}{l_{t}}\right) \\
& -2 l_{h}\left(l_{h}\left(R_{x}+R_{p}\right)\left(R_{b}+R_{a}\right) a^{2}\right. \\
& +\left(\left(\left(\frac{1}{2}\right)\left(3\left(R_{x}+R_{p}\right)\right) R_{b}-\left(\frac{1}{2}\right) R_{x} R_{p}\right) R_{a}-\left(\frac{1}{2}\right) R_{x} R_{p} R_{b}\right) l_{h} \\
& \left.\left.+\left(\left(\left(R_{x}+R_{p}\right) R_{b}+R_{x} R_{p}\right) R_{a}+R_{x} R_{p} R_{b}\right) l_{t}\right) a-l_{h} R_{x} R_{p} R_{b} R_{a}\right) \\
& \beta=\frac{1}{\Omega}\left(\left(2\left(R_{x}+R_{p}\right) R_{a} l_{h}^{2}+2\left(R_{x}+R_{p}\right) R_{a} l_{t} l_{h}\right) a \ln \left(\frac{l_{t}+l_{h}}{l_{t}}\right)\right. \\
& \left.-2 l_{h}\left(\left(R_{x}+R_{p}\right) R_{a} l_{h} a-l_{h} R_{x} R_{p} R_{a}\right)\right) \\
& \gamma=\frac{1}{\Omega}\left(2\left(R_{x}+R_{p}\right)\left(-R_{a}-R_{b}\right) l_{t} l_{h} \operatorname{aln}\left(\frac{l_{t}+l_{h}}{l_{t}}\right)\right. \\
& \left.-2 l_{h}\left(\left(\left(-R_{p}-R_{x}\right) R_{a}-\left(R_{p}+R_{x}\right) R_{b}\right) l_{h} a-l_{h}\left(R_{x}+R_{p}\right) R_{b} R_{a}\right)\right) \\
& \delta=\frac{1}{\Omega}\left(\left(2\left(R_{x}+R_{p}\right) R_{b} l_{h}^{2}+2\left(R_{x}+R_{p}\right) R_{b} l_{t} l_{h}\right) \operatorname{aln}\left(\frac{l_{t}+l_{h}}{l_{t}}\right)\right. \\
& \left.-2 l_{h}\left(\left(R_{p}+R_{x}\right) R_{b} l_{h} a-l_{h} R_{x} R_{p} R_{b}\right)\right) \\
& \Omega=2 l_{h}^{2}\left(a\left(R_{x}+R_{p}\right)\left(R_{b}+R_{a}\right) \ln \left(\frac{l_{t}+l_{h}}{l_{t}}\right)+\left(\left(R_{x}+R_{p}\right) R_{b}+R_{x} R_{p}\right) R_{a}+R_{x} R_{p} R_{b}\right)
\end{aligned}
$$

\section{S4. SEM of the probe}

Scanning Electron Microscopy images of the probe were recorded to confirm its dimensions. We also noted the effect of high current on the Pd film. Electron migrations limit the maximum current in the film which otherwise burns. 


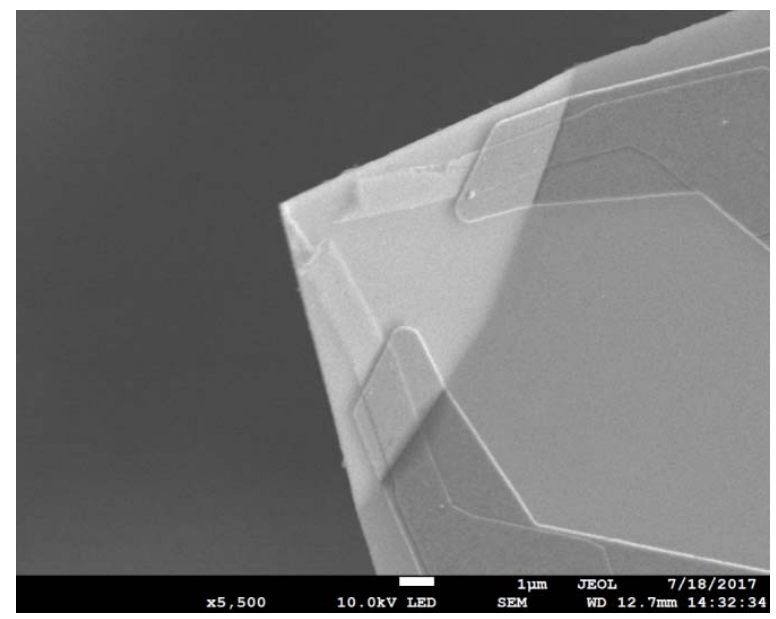

Fig. S3. SEM of the probe. It can be observed that the Pd film was burnt during experiments due to high current.

\section{S5. Temperature rise for different electrical resistances}

We computed the temperature arising from different electrical resistance in two cases: hotaverage heater temperature and tip temperature. We observe strong variations between these cases which are often considered equal.

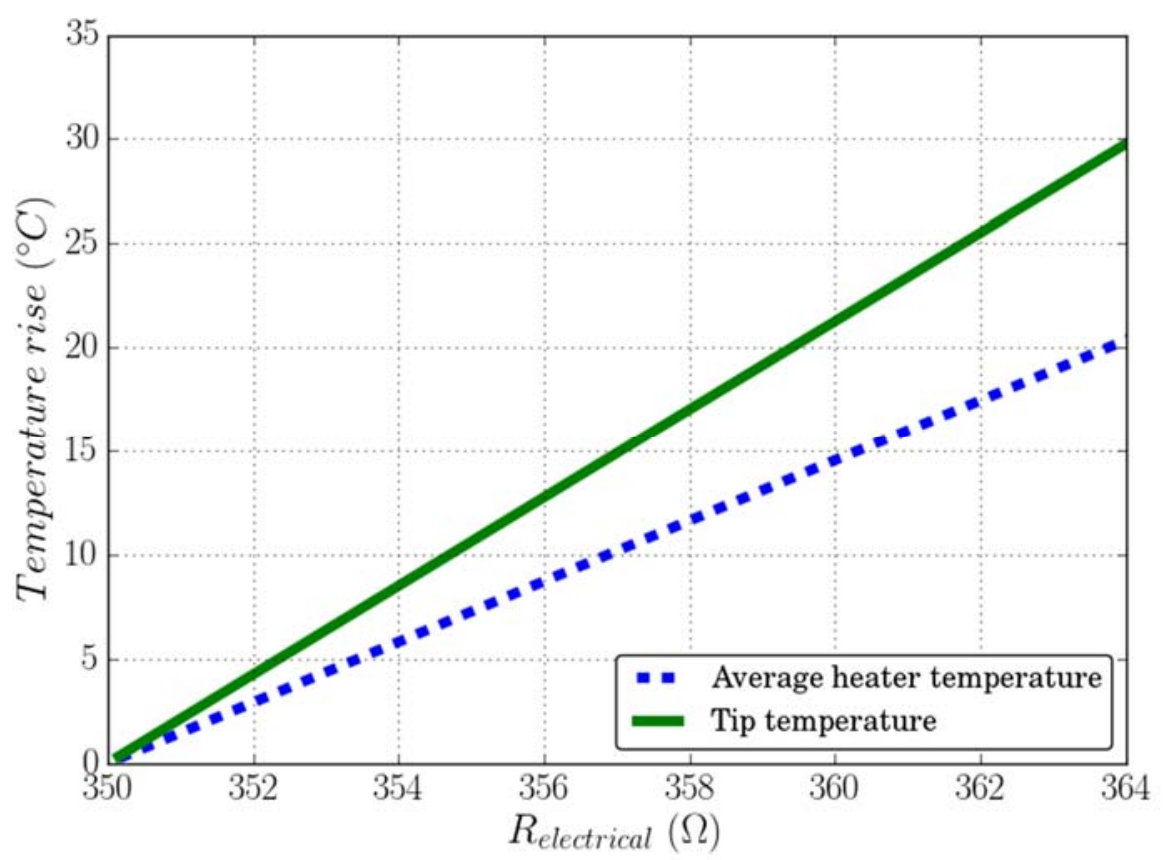

Fig. S4. Temperature rise dependence on the electrical resistance for the average heater temperature and the tip temperature. 
S6. Link between the proximity thermal resistance and the average heater temperature

We measure the proximity resistance by comparing the signal far away from the sample and just before the snap in contact. This way, it is possible to obtain $R_{p}$.

Far away from the sample, $\widehat{T}_{h}^{\infty}$ is given by

$$
\widehat{T}_{h}^{\infty}=\left[a\left(R_{B}+R_{\text {air }}\right) \ln \left(1+\frac{l_{h}}{l_{t}}\right)+\left(\frac { l _ { h } } { 2 l _ { t } } \left(\frac{\left(\left(2 R_{B}+a\right) R_{a i r}+R_{B} a\right) l_{h}}{l_{t}}-2 a\left(R_{B}+\right.\right.\right.\right.
$$

$\left.\left.\left.\left.R_{\text {air }}\right)\right)\right)\right] \frac{l_{t}^{2}}{\left(R_{B}+R_{\text {air }}\right) l_{h}^{2}} Q_{h}+\frac{R_{\text {air }}}{\left(R_{B}+R_{\text {air }}\right)} T_{M}+\frac{R_{B}}{\left(R_{B}+R_{\text {air }}\right)} T_{\text {air }}$

while just before snap in, it is given by

$$
\widehat{T}_{h}^{n c}=\alpha^{n c} Q_{h}+\beta^{n c} T_{M}+\gamma^{n c} T_{s}+\delta^{n c} T_{a i r}
$$

where

$$
\begin{aligned}
& \alpha^{n c} \\
& =\left(2 a \left(\left(\frac{a\left(R_{B}+R_{\text {air }}\right)}{2}+R_{-} B * R_{-} \text {air }\right) l_{h}^{2}+\left(a\left(R_{B}+R_{\text {air }}\right)+2 R_{B R_{\text {air }}}\right) l_{t} l_{h}\right.\right. \\
& \left.+\left(\left(R_{p}+R_{B}\right) R_{a i r}+R_{B R_{p}}\right) l_{t}^{2}\right) \ln \left(\frac{l_{t}+l_{h}}{l_{t}}\right) \\
& -\left(2\left(\left(R_{B}+R_{\text {air }}\right) a^{2}+\left(\left(\frac{3 R_{B}}{2}-\frac{R_{p}}{2}\right) R_{\text {air }}-\frac{R_{B} R_{p}}{2}\right) a-R_{\text {air }} R_{B} R_{p}\right) l_{h}\right. \\
& +a\left(\left(R_{p}+R_{B}\right) R_{a i r}\right. \\
& \left.\left.\left.+R_{B} R_{p}\right) l_{t}\right) l_{h}\right) \frac{Q_{h}}{\left(2\left(a\left(R_{B}+R_{\text {air }}\right) \ln \left(\frac{l_{t}+l_{h}}{l_{t}}\right)+\left(R_{p}+R_{B}\right) R_{a i r}+R_{B} R_{p}\right)\right) l_{h}^{2}}
\end{aligned}
$$




$$
\begin{gathered}
\beta^{n c}=\frac{2 a\left(R_{\text {air }} l_{h}^{2}+R_{\text {air }} l_{h} l_{t}\right) \ln \left(\frac{l_{t}+l_{h}}{l_{t}}\right)-\left(2\left(-R_{\text {air }} R_{p}+R_{\text {air }} a\right)\right) l_{h}^{2}}{\left(2\left(a\left(R_{B}+R_{\text {air }}\right) \ln \left(\frac{l_{t}+l_{h}}{l_{t}}\right)+\left(R_{p}+R_{B}\right) R_{\text {air }}+R_{B} R_{p}\right)\right) l_{h}^{2}} \\
\gamma^{n c}=\frac{2 a\left(-R_{\text {air }}-R_{B}\right) l_{t} l_{h} \ln \left(\frac{l_{t}+l_{h}}{l_{t}}\right)-\left(2\left(a\left(-R_{\text {air }}-R_{B}\right)-R_{B} R_{\text {air }}\right)\right) l_{h}^{2}}{\left(2\left(a\left(R_{B}+R_{\text {air }}\right) \ln \left(\frac{l_{t}+l_{h}}{l_{t}}\right)+\left(R_{p}+R_{B}\right) R_{\text {air }}+R_{B} R_{p}\right)\right) l_{h}^{2}} \\
\delta^{n c}=\frac{2 a\left(R_{B l_{h}^{2}}+R_{B} l_{h} l_{t}\right) \ln \left(\frac{l_{t}+l_{h}}{l_{t}}\right)-\left(2\left(-R_{B} R_{p}+R_{B} a\right)\right) l_{h}^{2}}{\left(2\left(a\left(R_{B}+R_{\text {air }}\right) \ln \left(\frac{l_{t}+l_{h}}{l_{t}}\right)+\left(R_{p}+R_{B}\right) R_{\text {air }}+R_{B} R_{p}\right)\right) l_{h}^{2}}
\end{gathered}
$$

